PROCEEDINGS OF THE

AMERICAN MATHEMATICAL SOCIETY

Volume 137, Number 6, June 2009, Pages 2015-2025

S 0002-9939(09)09734-2

Article electronically published on January 21, 2009

\title{
ELECTRICAL RESPONSE MATRIX OF A REGULAR 2n-GON
}

\author{
NATHANIEL D. BLAIR-STAHN AND DAVID B. WILSON \\ (Communicated by Edward C. Waymire)
}

\begin{abstract}
Consider a unit-resistive plate in the shape of a regular polygon with $2 n$ sides, in which even-numbered sides are wired to electrodes and oddnumbered sides are insulated. The response matrix, or Dirichlet-to-Neumann map, allows one to compute the currents flowing through the electrodes when they are held at specified voltages. We show that the entries of the response matrix of the regular $2 n$-gon are given by the differences of cotangents of evenly spaced angles, and we describe some connections with the limiting distributions of certain random spanning forests.
\end{abstract}

\section{INTRODUCTION}

Let $n \geq 2$ be an integer, and let $\mathcal{P}$ be a regular polygon with $2 n$ sides, centered at the origin in $\mathbb{C}$, with the midpoint of the $j^{\text {th }}$ side of $\mathcal{P}$ located on the unit circle at $e^{i \pi j / n}$ for $1 \leq j \leq 2 n$. We imagine $\mathcal{P}$ to be made of a unit resistive material, and we wish to determine how much current will flow through $\mathcal{P}$ when the even-numbered sides of $\mathcal{P}$ are wired to electrodes at specified voltages while the odd-numbered sides of $\mathcal{P}$ are kept insulated.

We will call the even-numbered (wired) sides of $\mathcal{P}$ nodes and the odd-numbered (insulated) sides free edges. Current may pass through the nodes, but no current is allowed to enter or exit $\mathcal{P}$ through the free edges. We number the $n$ nodes so that node $j$ corresponds to side $2 j$ of $\mathcal{P}$. For each $j$, the $j^{\text {th }}$ node of $\mathcal{P}$ is wired to an electrode and held at some specified voltage $v_{j}$. Each voltage configuration $\vec{v}=\left(v_{1}, \ldots, v_{n}\right)$ on the nodes results in some current output $\vec{I}=\left(I_{1}, \ldots, I_{n}\right)$, where $I_{j}$ is the current entering $\mathcal{P}$ through node $j$.

This problem can be rephrased in terms of the solution to a mixed DirichletNeumann boundary problem on the domain $\mathcal{P}$ : Given constants $v_{1}, \ldots, v_{n}$, there is a unique continuous function $V$ (the electric potential) on $\mathcal{P}$ which is harmonic on the interior, equals $v_{j}$ on side $2 j$, and has zero normal derivative on the free edges (see, e.g., Gam01, pg. 452 or pg. 445]). The current $I_{j}$ entering $\mathcal{P}$ through node $j$ is defined to be the flux of the electric field $E_{V}=-\nabla V$ through side $2 j$ into $\mathcal{P}$.

Since the current output $\vec{I}$ depends linearly on the voltage configuration $\vec{v}$, there is an $n \times n$ matrix $\Lambda$ mapping the space of voltage configurations to the space of current outputs. $\Lambda$ is called the response matrix or Dirichlet-to-Neumann map for the "resistor network" represented by $\mathcal{P}$. The Dirichlet-to-Neumann map $\Lambda$ is defined analogously for more general resistive domains or for resistor networks

Received by the editors April 3, 2007, and, in revised form, May 1, 2008.

2000 Mathematics Subject Classification. Primary 31A25; Secondary 30C20, 82B20, 05C05.

(C)2009 American Mathematical Society Reverts to public domain 28 years from publication 
represented by finite graphs (see, e.g., CdV98, IM98, CIM98, for background). Since, for any resistor network, the $k^{\text {th }}$ column of $\Lambda$ is the action of $\Lambda$ on the $k^{\text {th }}$ standard basis vector, $\Lambda_{j, k}$ will be the current entering the network ( $\mathcal{P}$ in our case) through node $j$ when node $k$ is held at 1 volt and all other nodes are held at 0 volts. Observe that $\Lambda_{j, k}$ will be positive if $j=k$ and negative otherwise. Although it is not obvious from this description, the response matrix for a general electrical network is symmetric (see CdV98). In this paper we compute the response matrix $\Lambda$ for $\mathcal{P}$ with the boundary conditions described above:

Theorem 1.1. The response matrix $\Lambda$ for the regular $2 n$-gon with alternating wired/free boundary conditions has entries given by

$$
\Lambda_{j, k}=\frac{\cot \left[\frac{\pi}{n}\left(j-k+\frac{1}{2}\right)\right]-\cot \left[\frac{\pi}{n}\left(j-k-\frac{1}{2}\right)\right]}{n} .
$$

The response matrix is closely connected to the distribution of random "groves" (a generalization of spanning trees) in a resistor network KW06. In $₫ 2$ we give some background on this connection with groves, which is what initially led us to study the response matrix $\Lambda$, and we briefly compare the grove model with an analogous model based on percolation. We then discuss a purely algebraic approach (based on groves) that Kenyon and Wilson KW06, §5.2] used to compute the response matrix for the regular $2 n$-gon in the cases $n=3$ and $n=4$. This algebraic approach, however, is not easily adapted to general $n$. We prove Theorem 1.1 in $₫ 3$ using a combination of algebraic and analytic methods.

We mention that it is known how to compute the response matrix by using the Schwarz-Christoffel formula to map the polygon to a rectangle with vertical slits that correspond to the free edges, as shown in Figure 1. A general $2 n$-gon may be conformally mapped to a rectangle so that one wired side (say side $j$ ) gets mapped to the top of the rectangle, the adjacent free sides get mapped to the sides of the rectangle, the remaining wired sides get mapped to intervals of the bottom side of the rectangle, and the remaining free sides get mapped to vertical slits. ([KW06. $\S 5.2]$ includes a discussion of these maps.) The current response $\Lambda_{j, k}$ is just the ratio of the length of the image of the $k^{\text {th }}$ side to the height of the rectangle. Without going further into details, we mention that in the example of the regular octagon $(n=4)$, this approach yields

$$
\Lambda_{j, j+2}=-\frac{\int_{x_{4}}^{x_{5}} \frac{w^{2}-b^{2}}{\prod_{\ell=1}^{8}\left(w-x_{\ell}\right)^{1 / 2}} d w}{\int_{x_{7}}^{x_{8}} \frac{w^{2}-b^{2}}{\prod_{\ell=1}^{8}\left(w-x_{\ell}\right)^{1 / 2}} d w} \quad \text { where } \quad b^{2}=\frac{\int_{x_{3}}^{x_{4}} \frac{w^{2}}{\prod_{x_{3}}^{8}\left(w-x_{\ell}\right)^{1 / 2}} d w}{\int_{x_{4}}^{x_{\ell=1}\left(w-x_{\ell}\right)^{1 / 2}} d w}
$$

and $x_{\ell}=\cot ((1 / 2-\ell) \pi / 8)$. It is not at first obvious that this should simplify to

$$
\Lambda_{j, j+2}=1 / 2-1 / \sqrt{2}
$$

Remark 1.2. Since the current responses for the regular polygon are differences of cotangents, the horizontal positions of the vertical slits and the sides of the rectangle are given by cotangents of evenly spaced angles. If we view the slit rectangle as being embedded in the upper half-plane with the rectangle's bottom edge on the real axis, then there is a Möbius transformation of the upper half-plane to the unit 

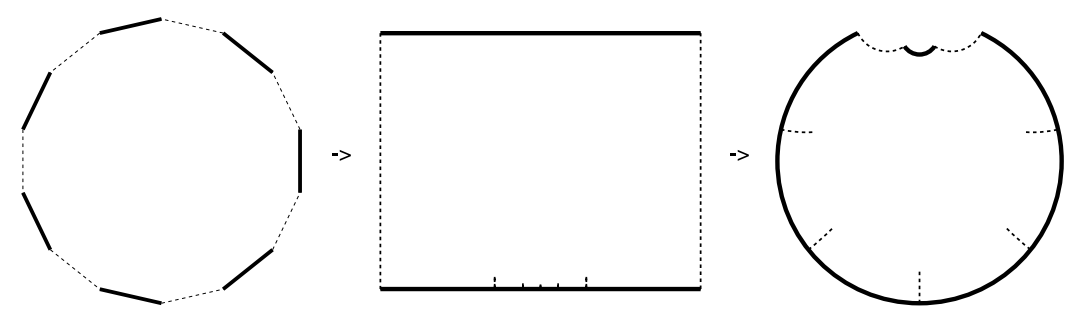

FiguRE 1 . On the left is the regular $2 n$-gon $(n=7)$ with alternate sides wired and free (insulated). The regular $2 n$-gon is conformally mapped to a rectangle so that one wired side goes to the top and the remaining wired sides go the bottom of the rectangle, while two free sides get mapped to the sides of the rectangle and the remaining free sides get mapped to vertical slits. Entries of the response matrix are given by the lengths of the wired sides in the rectangle divided by the height of the rectangle. A Möbius transformation maps the slit rectangle to a subset of the unit disk. Each wired side and each free side is mapped to an arc of a circle that passes through the top of the disk, and $n-1$ of the wired sides each get mapped to arcs of the unit circle of length $2 \pi / n$.

disk so that images of the bases of the vertical slits (and the two bottom corners) are evenly spaced on the unit circle (Figure 1).

\section{The RESPONSE MATRIX AND RANDOM GROVES}

We give here some background on the relation between the response matrix of a graph and random "groves" of that graph. A grove is a forest, i.e., an acyclic collection of edges of the graph, such that every constituent tree of the forest contains at least one of a special set of distinguished vertices, which are called nodes. The upper-left panel of Figure 2 shows a grove on a graph with two nodes (labeled 1 and 2), and the lower-left panel shows a grove on a graph with three nodes (labeled 1,2 , and 3). The first grove consists of one tree, which contains the two nodes 1 and 2 , and the path connecting the nodes is highlighted. The second grove consists of two trees, one of which contains nodes 1 and 3 , while the other contains node 2 .

Groves of "circular planar graphs" arise naturally in combinatorics CS04 and statistical physics KW06. A graph with distinguished nodes is "circular planar" if it embeds in the plane and each of the nodes lies on the outer face; the nodes are numbered in a counterclockwise order. If a uniformly random grove is chosen, this defines a random (noncrossing) partition $\sigma$ on the set of nodes, and we are interested in the probability distribution of this random partition.

Another natural probability distribution on node partitions arises from percolation on the graph (where each edge is included independently with probability $p$ ); this is illustrated in the right-hand panels of Figure 2 for $p=1 / 2$. There has been significant recent work in studying crossing events for percolation such as those shown in Figure 2, and it is interesting to compare the crossing event probabilities in these two models. 
For graphs with two nodes, the partition probabilities for groves follow from Kirchhoff's formula [Kir90]:

$$
\frac{\# \text { spanning trees }}{\# \text { two-tree forests with }}=\frac{\operatorname{Pr}[\text { grove partition is } 12]}{\operatorname{Pr}[\text { grove partition is } 1 \mid 2]}=\frac{1}{R_{1,2}}=-\Lambda_{1,2}
$$

where $R_{1,2}$ is the electrical resistance between nodes 1 and 2 when the graph is viewed as a resistor network in which each edge has unit resistance. (The relation $\Lambda_{1,2}=-1 / R_{1,2}$ holds for two nodes only; when there are more nodes, the response matrix entries are more complicated functions of the pairwise resistances.)

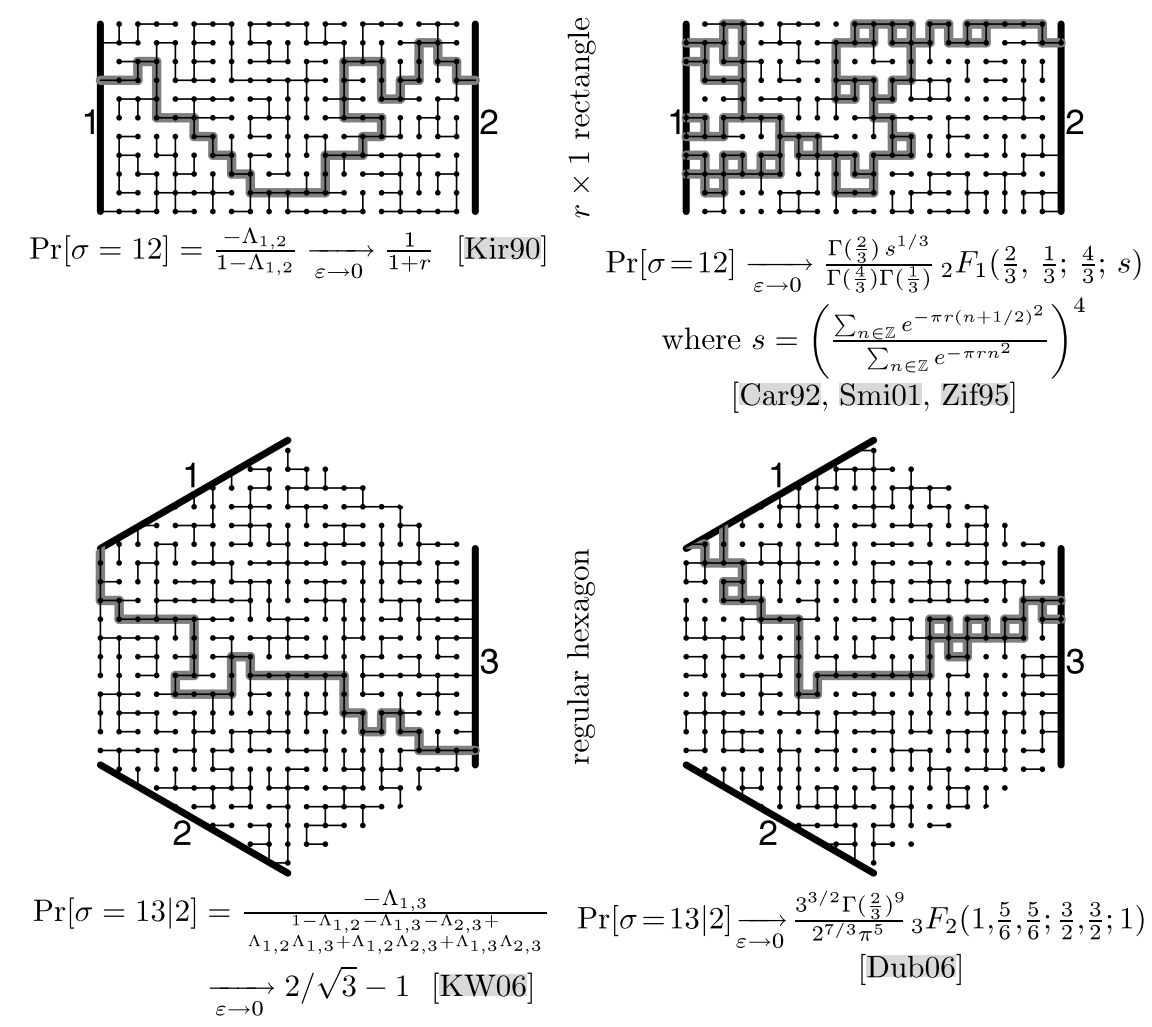

Figure 2. Examples of grove (left) and percolation (right) configurations on regions with two (upper) or three (lower) nodes. Random groves and percolation are both important special cases of the Fortuin-Kasteleyn random cluster model of statistical physics FK72. The paths connecting the nodes are highlighted, and when the grid spacing $\varepsilon$ tends to 0 , the probability that the nodes are connected in a given way is indicated below the panels. (The percolation formulas are rigorous for site percolation on the triangular lattice and are believed to hold for bond percolation on the square lattice as shown here.) 
In the case where the graph is a fine grid restricted to an $r \times 1$ rectangular region, with the two nodes being extra vertices corresponding to the left and right edges of the rectangle (as illustrated in Figure 2), then $R_{1,2}=r$, so the probability that the grove partition $\sigma$ is 12 is simply $\operatorname{Pr}[\sigma=12]=1 /(1+r)$. If the fine grid is restricted to a region different from a rectangle, with two nodes each occupying some fraction of the boundary, then the region may be conformally mapped to a rectangle of some aspect ratio $r$, with the two nodes getting mapped to the left and right edges. When the grid becomes very fine, the resistance between the nodes in the original domain converges to $r$, so in this limit $\operatorname{Pr}[\sigma=12] \rightarrow 1 /(1+r)$.

The corresponding formula for critical percolation (where each edge occurs independently with probability $1 / 2$ ) was deduced by Cardy Car92 (see upper-right panel of Figure 2) using exact but nonrigorous methods. (Ziff adapted Cardy's formula, which was given for the upper half-plane, to rectangular regions [Zif95], and Smirnov gave a rigorous proof for the related model of site percolation Smi01.)

More recently these boundary crossing events have been studied in regions with more nodes [Dub06, KW06; this is illustrated in the lower-right (for percolation) and lower-left (for groves) panels of Figure 2. In the case of groves, Kenyon and Wilson KW06 show how to compute the boundary partition probabilities for any number of nodes in terms of the entries of the response matrix $\Lambda$ of the graph when viewed as an electrical network. It is convenient to abbreviate

$$
\dddot{\operatorname{Pr}}(\sigma)=\frac{\operatorname{Pr}[\text { grove partition is } \sigma]}{\operatorname{Pr}[\text { grove partition is } 1|2| \ldots \mid n]} .
$$

For graphs with three nodes the analogues of Kirchhoff's formula (2.1) are

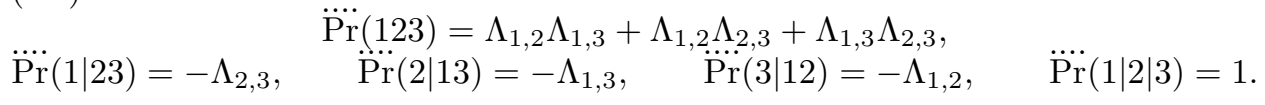

More generally, Kenyon and Wilson KW06 proved that for any circular planar graph with any number $n$ of nodes on the outer face, if $\sigma$ is any noncrossing partition on $\{1, \ldots, n\}$, then $\dddot{\operatorname{Pr}}(\sigma)$ is a polynomial in the entries of the response matrix $\Lambda$ and can be computed explicitly.

It is interesting to see what these general formulas give for some nice special cases. For example, Dubédat Dub06] computed the partition probabilities for (site) percolation when the region is the regular hexagon with 3 nodes along alternate sides of the hexagon. (The lower-right panel of Figure 2 illustrates this for bond percolation.) To carry out a similar computation for groves, we need to calculate the response matrix for the regular hexagon with alternating free and wired boundary conditions on its faces.

In the introduction we mentioned an algebraic approach for computing the response matrix $\Lambda_{j, k}$ for a regular $2 n$-gon. This approach [KW06, §5.2] is based on enumerating trees in a random grove; we briefly describe this tree-enumeration approach together with some associated open problems.

We consider random groves on a very fine grid restricted to the regular $2 n$-gon, with $n$ nodes "wired" to every other side of the polygon (as in Figure 2). Using the formulas from [KW06], we may express the polynomial

$$
P_{n}(q)=\sum_{t=1}^{n} \frac{\operatorname{Pr}[t \text { trees in grove }]}{\operatorname{Pr}[n \text { trees in grove }]} q^{t-1}
$$


in terms of the response matrix of the graph, which in the scaling limit approaches the response matrix $\Lambda$ of the regular $2 n$-gon. Since (by symmetry considerations) the response matrix for the regular $2 n$-gon is circulant, we may define $\Lambda_{|j-k|}=\Lambda_{j, k}$ (where indices are identified $\bmod n$ ). From the above formulas (2.2) we see that, in the limit, $P_{3}(q)=1-3 \Lambda_{1} q+3 \Lambda_{1}^{2} q^{2}$. For the regular octagon it turns out that

$$
P_{4}(q)=1-q\left(4 \Lambda_{1}+2 \Lambda_{2}\right)+q^{2}\left(6 \Lambda_{1}^{2}+8 \Lambda_{1} \Lambda_{2}+2 \Lambda_{2}^{2}\right)-q^{3}\left(4 \Lambda_{1}^{3}+8 \Lambda_{1}^{2} \Lambda_{2}+4 \Lambda_{1} \Lambda_{2}^{2}\right) .
$$

For each grove of a circular planar graph with $n$ nodes there is a dual grove on the dual graph, which contains the duals of edges not contained in the primal grove. The number of trees in a grove plus the number of trees in its dual grove is $n+1$. Since the dual graph of a fine grid restricted to the regular $2 n$-gon with alternate wired/free boundary conditions is again a fine grid restricted to the regular $2 n$ gon with alternate free/wired boundary conditions, in the limit where the grid is very fine, the probability of seeing $t$ trees in a random grove equals the probability of seeing $n-t+1$ trees. Hence the coefficients of the polynomial $P_{n}(q)$ form a palindrome. For $n=3$ this implies that $3 \Lambda_{1}^{2}=1$, or $\Lambda_{1}=-1 / \sqrt{3}$, determining the response matrix. For $n=4$ there are several pairs $\left(\Lambda_{1}, \Lambda_{2}\right)$ which make $P_{4}(q)$ a palindrome, but only one in which $\Lambda_{1}$ and $\Lambda_{2}$ are both negative $\left(\Lambda_{1}=-1 / 2\right.$, $\left.\Lambda_{2}=1 / 2-1 / \sqrt{2}\right)$, which is enough to determine the response matrix. For general $n$ the fact that $P_{n}(q)$ is a palindromic polynomial generates enough constraints to limit the coefficients of $\Lambda$ to a zero-dimensional algebraic variety, but it is not clear that there will always be a unique negative solution for the $\Lambda$ 's, nor is it clear how to obtain the solution for general $n$ using this algebraic approach.

However, in the next section we explicitly compute the response matrix for the polygon $\mathcal{P}$ by other means, proving Theorem 1.1. Combining this result with the formulas from [KW06], we may write down the first few polynomials $P_{n}(q)$ :

$$
\begin{aligned}
& P_{2}(q)=1+q \\
& P_{3}(q)=1+\sqrt{3} q+q^{2} \\
& P_{4}(q)=1+(1+\sqrt{2}) q+(1+\sqrt{2}) q^{2}+q^{3} \\
& P_{5}(q)=1+\sqrt{5+2 \sqrt{5}} q+(2+\sqrt{5}) q^{2}+\sqrt{5+2 \sqrt{5}} q^{3}+q^{4} \\
& P_{6}(q)=1+(2+\sqrt{3}) q+(3+2 \sqrt{3}) q^{2}+(3+2 \sqrt{3}) q^{3}+(2+\sqrt{3}) q^{4}+q^{5} .
\end{aligned}
$$

The constant term is of course always 1. Referring to KW06, the linear term is $-\sum_{j<k} \Lambda_{j, k}$, which simplifies to $\cot (\pi /(2 n))$. It would be interesting to better understand the polynomials $P_{n}$, such as for example the approximate distribution of the number of trees for large $n$, or the asymptotics of

$$
P_{n}(1)=1 / \operatorname{Pr}[\text { random grove is a single tree]. }
$$

\section{Computing $\Lambda$}

This section is devoted to proving Theorem 1.1. In $\$ 3.1$ we identify the eigenvectors and eigenvalues of $\Lambda$ using symmetry considerations, thereby finding a diagonalization of $\Lambda$. Then in $\$ 3.2$ we compute $\Lambda$ by performing a matrix multiplication to change from the eigenbasis back to the standard basis. 
3.1. Complex potential and the diagonalization of $\Lambda$. The key to finding the eigenvectors and eigenvalues of $\Lambda$ is to introduce complex electric potentials and currents in order to exploit the symmetry of the polygon $\mathcal{P}$. A complex-valued potential $V$ on $\mathcal{P}$ can be thought of as two separate real potentials, one from the real part of $V$ and the other from the imaginary part. The electric field $E_{V}$ now takes values in $\mathbb{C}^{2}$ rather than $\mathbb{R}^{2}$, and can be thought of as carrying separate real and imaginary currents. The current output $\vec{I}$ will now be a complex-linear function of the voltage configuration $\vec{v}$. Thus we can view $\Lambda$ as a complex-linear transformation of $\mathbb{C}^{n}$ whose restriction to $\mathbb{R}^{n}$ yields the expected real current outputs.

Let $\omega=e^{2 \pi i / n}$, and for $1 \leq k \leq n$, define the voltage configuration $\vec{v}_{k}$ by $\left(\vec{v}_{k}\right)_{j}=\omega^{j k}$.

Lemma 3.1. Let $1 \leq k \leq n$. Then $\vec{v}_{k}=\left(\omega^{k}, \omega^{2 k}, \ldots, \omega^{(n-1) k}, 1\right)$ is an eigenvector of $\Lambda$, and the corresponding eigenvalue $\lambda_{k}$ is the current entering $\mathcal{P}$ through node $n$ under the voltage configuration $\vec{v}_{k}$.

Proof. Let $\sigma: \mathbb{C}^{n} \rightarrow \mathbb{C}^{n}$ denote the function which cyclically shifts the components of a vector to the left: $\sigma\left(v_{1}, v_{2}, \ldots, v_{n-1}, v_{n}\right)=\left(v_{2}, v_{3}, \ldots, v_{n}, v_{1}\right)$. If $\vec{v}$ is a voltage configuration on $\mathcal{P}, \sigma \vec{v}$ is the voltage configuration obtained by replacing the voltage on node $j$ with the voltage on node $j+1$ (where the indices are identified mod $n$ ). Because $\mathcal{P}$ is rotationally symmetric, the resulting currents will likewise be rotated clockwise by one node. That is,

$$
\Lambda(\sigma \vec{v})=\sigma(\Lambda \vec{v}) \text { for all } \vec{v} \in \mathbb{C}^{n} .
$$

On the other hand, for each vector $\vec{v}_{k}=\left(\omega^{k}, \omega^{2 k}, \ldots, \omega^{(n-1) k}, 1\right)$, we have $\sigma \vec{v}_{k}=$ $\omega^{k} \vec{v}_{k}$. If $\vec{I}_{k}=\Lambda \vec{v}_{k}$ is the current output resulting from $\vec{v}_{k}$, then we have

$$
\sigma \vec{I}_{k}=\sigma\left(\Lambda \vec{v}_{k}\right)=\Lambda\left(\sigma \vec{v}_{k}\right)=\Lambda\left(\omega^{k} \vec{v}_{k}\right)=\omega^{k} \vec{I}_{k} .
$$

This implies $\left(\vec{I}_{k}\right)_{j}=\omega^{j k}\left(\vec{I}_{k}\right)_{n}$, so $\Lambda \vec{v}_{k}=\vec{I}_{k}=\left(\vec{I}_{k}\right)_{n} \vec{v}_{k}$.

Next we compute the eigencurrent $\lambda_{k}=\left(\vec{I}_{k}\right)_{n}$ by considering the harmonic conjugate of the potential induced by $\vec{v}_{k}$. Since harmonic conjugation defines a real-linear operator - call it $\mathcal{H}$ - on real-valued harmonic functions on $\mathcal{P}$ (modulo constant functions), there is a unique way to extend $\mathcal{H}$ to a complex-linear operator on complex-valued harmonic functions on $\mathcal{P}$. Recall that any harmonic conjugate satisfies the Cauchy-Riemann equations,

$$
(\mathcal{H} V)_{x}=-V_{y} \text { and }(\mathcal{H} V)_{y}=V_{x},
$$

so that $\nabla \mathcal{H} V$ is orthogonal to $\nabla V$ at every point. The Cauchy-Riemann equations imply the following well-known result (see, e.g., Gam01, Section III.6]), which we shall use to compute the current $\lambda_{k}$ :

Proposition 3.2. Let $\Omega \subseteq \mathbb{C}$ be simply connected, let $V: \Omega \rightarrow \mathbb{C}$ be harmonic, and let $\gamma:[0,1] \rightarrow \Omega$ be a $C^{1}$ path with $\gamma(0)=a$ and $\gamma(1)=b$. If $\mathcal{H} V$ is any harmonic conjugate of $V$, then the current due to $V$ flowing from left to right across $\gamma$ is equal to $\mathcal{H} V(a)-\mathcal{H} V(b)$ (where 'left' and 'right' are defined relative to $\gamma$ 's orientation).

Now let $\mathcal{H}_{0} V$ denote the harmonic conjugate of $V$ satisfying $\left(\mathcal{H}_{0} V\right)(0)=i V(0)$, so that $\mathcal{H}_{0}^{2}=-\mathrm{Id}$, and let $V_{k}: \mathcal{P} \rightarrow \mathbb{C}$ be the potential function induced by the voltage configuration $\vec{v}_{k}$. 
Lemma 3.3. Let $\zeta=e^{i \pi / n}\left(\right.$ so $\left.\zeta^{2}=\omega\right)$. Then $\mathcal{H}_{0} V_{k}$ takes the value $-i \zeta^{(2 j-1) k}$ on side $2 j-1$, for $1 \leq j \leq n$, and has zero normal derivative on the even-numbered sides of $\mathcal{P}$ (see Figure 3 ). Under the potential $V_{k}$, the current $\lambda_{k}$ entering $\mathcal{P}$ through side $2 n$ is $2 \sin (\pi k / n)$.

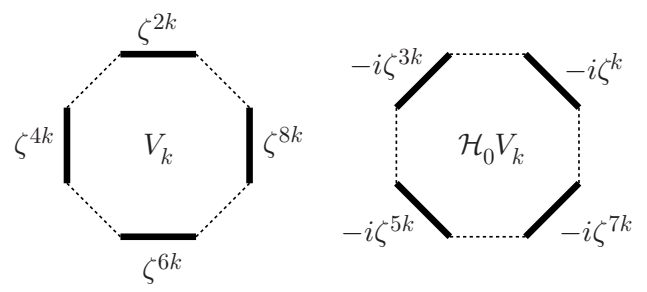

FiguRE 3. Boundary conditions of $V_{k}$ and $\mathcal{H}_{0} V_{k}$ for $n=4$, illustrating Lemma 3.3. Thick solid lines represent wired sides of $\mathcal{P}$, and thin dashed lines represent free sides.

Proof. The fact that the boundary conditions of $\mathcal{H}_{0} V_{k}$ are wired where $V_{k}$ is free and vice versa is immediate from the Cauchy-Riemann equations: Since $\nabla V_{k}$ is oriented parallel to the boundary on the free edges and orthogonal to the boundary on the nodes, the reverse is true for $\nabla \mathcal{H} V_{k}$. (Boundary issues can be dealt with by using Schwarz reflection to enlarge the domain, moving the boundary sides to the interior.)

Recall that rotating the voltages in the configuration $\vec{v}_{k}$ clockwise by one node is the same as multiplying them by $\omega^{k}$. Thus, if $z$ is on an even-numbered side of $\mathcal{P}$, then

$$
V_{k}(\omega z)=\omega^{k} V_{k}(z)
$$

Now for each $\alpha \in \mathbb{C}$ with $|\alpha|=1$, we define the rotation operator $R_{\alpha}(z)=\alpha z$ for $z \in \mathbb{C}$. With this notation, (3.1) says that the functions $V_{k} \circ R_{\omega}$ and $\omega^{k} V_{k}$ agree on the nodes of $\mathcal{P}$. Since they are also both continuous on $\mathcal{P}$, harmonic on the interior, and have zero normal derivative on the free edges, they must be equal, so (3.1) in fact holds for all $z \in \mathcal{P}$.

Since $\mathcal{H}$ is linear, (3.1) implies $\mathcal{H}_{0} V_{k}(\omega z)=\omega^{k} \mathcal{H}_{0} V_{k}(z)+C$ for some constant $C$. Setting $z=0$ shows that $C=\left(1-\omega^{k}\right) \mathcal{H}_{0} V_{k}(0)=0$ (because $\mathcal{H}_{0} V_{k}(0)=i V_{k}(0)=0$ if $k \neq n$, and $\left.1-\omega^{n}=0\right)$. Thus we have

$$
\mathcal{H}_{0} V_{k}(\omega z)=\omega^{k} \mathcal{H}_{0} V_{k}(z)
$$

for all $z \in \mathcal{P}$, so $\mathcal{H}_{0} V_{k}$ has the same type of rotational symmetry as $V_{k}$.

Let $a_{k}$ denote the (constant) value of $\mathcal{H}_{0} V_{k}$ on side 1 . Combining (3.2) with the fact that $\mathcal{H}_{0} V_{k}$ has alternating free/wired boundary conditions shows that the boundary conditions of the harmonic functions $a_{k} V_{k}$ and $\left(\mathcal{H}_{0} V_{k}\right) \circ R_{\zeta}$ agree, so these functions must be equal. Therefore,

$$
\begin{aligned}
\mathcal{H}_{0} V_{k} & =a_{k} V_{k} \circ R_{\zeta^{-1}}, \\
\mathcal{H}_{0}^{2} V_{k} & =a_{k} \mathcal{H}_{0}\left(V_{k} \circ R_{\zeta^{-1}}\right), \\
-V_{k} & =a_{k}\left(\mathcal{H}_{0} V_{k}\right) \circ R_{\zeta^{-1}}=a_{k}^{2} V_{k} \circ R_{\zeta^{-2}}=a_{k}^{2} \omega^{-k} V_{k},
\end{aligned}
$$

which shows that $a_{k}= \pm i \zeta^{k}$ (note that (3.1) was used in the last step). 
If $k=n$, then $V_{k} \equiv 1$, which implies $\mathcal{H}_{0} V_{k} \equiv i$, so $a_{n}=i=-i \zeta^{n}$ and $\lambda_{n}=0$. If $1 \leq k<n$, we use Proposition 3.2 to compute the current $\lambda_{k}$, up to sign:

$\lambda_{k}=\mathcal{H}_{0} V_{k}\left(e^{i \pi / 2 n}\right)-\mathcal{H}_{0} V_{k}\left(e^{-i \pi / 2 n}\right)=a_{k}-\omega^{-k} a_{k}= \pm i \zeta^{k} \mp i \zeta^{-k}=\mp 2 \sin (\pi k / n)$.

To determine the sign, note that since $V_{k}$ is harmonic, we have $V_{k}(z)=\mathbb{E}\left[V_{k}\left(z_{T}\right)\right]$ for all $z \in \mathcal{P}$, where $z_{t}$ is a standard Brownian motion started at $z_{0}=z$ which is reflected off the odd sides and absorbed at the even sides, and $T$ is the absorption time. On the even-numbered sides, $\operatorname{Re} V_{k}(z)$ is maximized (with value 1 ) on side $2 n$. Thus $\operatorname{Re} V_{k}(z)<1$ for $z$ in the interior of the polygon $\mathcal{P}$, so the real part of the electric field on side $2 n$ points into $\mathcal{P}$. Hence the real part of the current entering $\mathcal{P}$ on side $2 n$ must be nonnegative, so it is $+2 \sin (\pi k / n)$, and $a_{k}=-i \zeta^{k}$.

3.2. Recovering $\Lambda$ from its diagonalization. Putting together Lemmas 3.1 and 3.3 we have

Theorem 3.4. The response matrix $\Lambda$ satisfies $\Lambda W=W D$, where $W$ is the matrix of eigenvectors given by $W_{j, k}=e^{2 \pi i j k / n}$, and $D$ is the diagonal matrix of eigenvalues with $D_{k, k}=\lambda_{k}=2 \sin (\pi k / n)$.

We use the following lemma to compute $\Lambda$ from Theorem 3.4 Recall that $\zeta=$ $e^{i \pi / n}$.

Lemma 3.5. If $m$ is any integer, then

$$
\sum_{\ell=1}^{n} \zeta^{(2 m \pm 1) \ell}=-1+i \cot \left[\frac{\pi}{n}\left(m \pm \frac{1}{2}\right)\right] .
$$

Proof. Let $b_{\ell}=\zeta^{(2 m \pm 1) \ell}$ and let $\beta=\sum_{\ell=1}^{n} b_{\ell}$. We first show that $\operatorname{Re} \beta=-1$. Since $b_{n-\ell}=-\overline{b_{\ell}}$, the real parts of $b_{\ell}$ and $b_{n-\ell}$ add to 0 (or the real part equals 0 if $\ell=n / 2$ ). Pairing up the terms in this way, we see that $\operatorname{Re} \sum_{\ell=1}^{n-1} b_{\ell}=0$ and hence $\operatorname{Re} \beta=\operatorname{Re} b_{n}=-1$.

Now let $\theta=\frac{\pi}{n}\left(m \pm \frac{1}{2}\right)$, and define $\beta^{\prime}=e^{-i \theta} \beta$. We will use the same trick as above to show that $\beta^{\prime}$ is imaginary, then show that this implies $\operatorname{Im} \beta=\cot \theta$ (see Figure (4). Note that $\beta^{\prime}=\sum_{\ell=1}^{n} c_{\ell}$, where $c_{\ell}=e^{-i \theta} b_{\ell}=e^{\frac{i \pi}{2 n}(2 m \pm 1)(2 \ell-1)}$. Now,

$$
c_{n+1-\ell}=e^{\frac{i \pi}{2 n}(2 m \pm 1)(2 n-2 \ell+1)}=e^{ \pm i \pi} e^{-\frac{i \pi}{2 n}(2 m \pm 1)(2 \ell-1)}=-\overline{c_{\ell}},
$$

so $c_{\ell}$ and $c_{n+1-\ell}$ are symmetric about the imaginary axis. It follows that $\beta^{\prime}$ is imaginary and hence $\arg \beta=\theta+\arg \beta^{\prime}=\theta \pm \frac{\pi}{2}$. Finally, observe that

$$
\operatorname{Im} \beta=\operatorname{Re} \beta \cdot \tan (\arg \beta)=-1 \cdot \tan \left(\theta \pm \frac{\pi}{2}\right)=\cot \theta=\cot \left[\frac{\pi}{n}\left(m \pm \frac{1}{2}\right)\right] .
$$

Proof of Theorem 1.1. First we observe that the matrix $W$ in Theorem 3.4 is invertible with $W^{-1}=\frac{1}{n} W^{*}$. (One can easily verify this directly, or simply notice that $\frac{1}{\sqrt{n}} W$ is the inverse discrete Fourier transform matrix, which is unitary.) We need to show that the entries of the matrix $W D W^{-1}$ agree with the formula for $\Lambda$ given in Theorem 1.1. It follows from Theorem 3.4 that $(W D)_{j, k}=W_{j, k} D_{k, k}=$ 


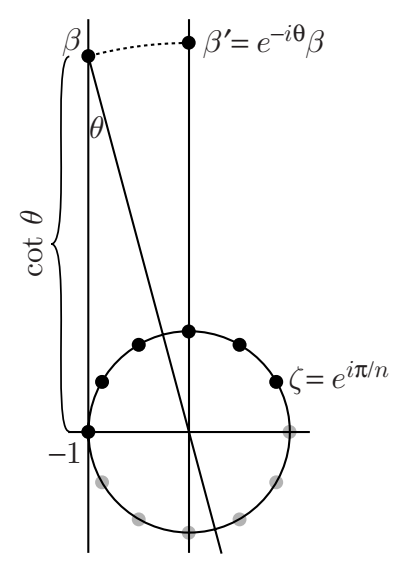

Figure 4. Illustration of the proof of Lemma 3.5 with $n=6$ and $m=0$. The first five powers of $\zeta$ are symmetric about the imaginary axis, so the $\operatorname{sum} \beta=\sum_{\ell=1}^{6} \zeta^{\ell}$ lies on the line $\operatorname{Re} z=-1$. The first six powers of $\zeta$ are symmetric about the line $\arg z=$ $\theta+\pi / 2$, where $\theta=\pi / 12$, so $\beta$ also lies on this line (equivalently, $\beta^{\prime}=e^{-i \theta} \beta$ is imaginary).

$$
\begin{aligned}
\zeta^{2 j k} \lambda_{k}=\zeta^{2 j k}\left(\zeta^{k}-\zeta^{-k}\right) / i \text {. Since }\left(W^{-1}\right)_{j, k}=\frac{1}{n} \overline{W_{k, j}}=\zeta^{-2 j k} / n, \text { we have } \\
\Lambda_{j, k}=\left(W D W^{-1}\right)_{j, k}=\sum_{\ell=1}^{n}(W D)_{j, \ell}\left(W^{-1}\right)_{\ell, k}=\frac{1}{i n} \sum_{\ell=1}^{n} \zeta^{2 j \ell}\left(\zeta^{\ell}-\zeta^{-\ell}\right) \zeta^{-2 \ell k} \\
=\frac{1}{i n} \sum_{\ell=1}^{n}\left[\left(\zeta^{2(j-k)+1}\right)^{\ell}-\left(\zeta^{2(j-k)-1}\right)^{\ell}\right] \\
=\frac{\cot \left[\frac{\pi}{n}\left(j-k+\frac{1}{2}\right)\right]-\cot \left[\frac{\pi}{n}\left(j-k-\frac{1}{2}\right)\right]}{n},
\end{aligned}
$$

where the last equality follows from Lemma 3.5

\section{ACKNowledgement}

We thank Richard Kenyon for helpful discussions, in particular for suggesting the use of complex voltages.

\section{REFERENCES}

[Car92] John L. Cardy, Critical percolation in finite geometries, J. Phys. A 25 (1992), no. 4, L201-L206. MR:1151081 (92m:82048)

[CdV98] Yves Colin de Verdière, Spectres de graphes, Cours Spécialisés [Specialized Courses], vol. 4, Société Mathématique de France, Paris, 1998. MR.1652692 (99k:05108)

[CIM98] E. B. Curtis, D. Ingerman, and J. A. Morrow, Circular planar graphs and resistor networks, Linear Algebra Appl. 283 (1998), no. 1-3, 115-150. MR1657214|(99k:05096)

[CS04] Gabriel D. Carroll and David Speyer, The cube recurrence, Electron. J. Combin. 11 (2004), no. 1, Research Paper 73, 31 pp. MR2097339 (2005f:05007)

[Dub06] Julien Dubédat, Euler integrals for commuting SLEs, J. Stat. Phys. 123 (2006), no. 6, 1183-1218. MR2253875(2007g:82027)

[FK72] C. M. Fortuin and P. W. Kasteleyn, On the random-cluster model. I. Introduction and relation to other models, Physica 57 (1972), 536-564. MR0359655 (50:12107) 
[Gam01] Theodore W. Gamelin, Complex Analysis, Undergraduate Texts in Mathematics, Springer-Verlag, New York, 2001. MR1830078 (2002h:30001)

[GM05] John B. Garnett and Donald E. Marshall, Harmonic Measure, New Mathematical Monographs, vol. 2, Cambridge University Press, 2005. MR2150803 (2006g:31002)

[IM98] David Ingerman and James A. Morrow, On a characterization of the kernel of the Dirichlet-to-Neumann map for a planar region, SIAM J. Math. Anal. 29 (1998), no. 1, 106-115 (electronic). MR:1617177 (99c:35258)

[Kir90] Gustav Kirchhoff, Beweis der Existenz des Potentials das an der Grenze des betrachteten Raumes gegebene Werthe hat für den Fall dass diese Grenze eine überall convexe Fläche ist, Acta Math. 14 (1890), no. 1, 179-183. MR.1554794

[KW06] Richard W. Kenyon and David B. Wilson, Boundary partitions in trees and dimers, 2006. To appear in Trans. Amer. Math. Soc. http://arxiv.org/abs/math.PR/0608422

[Smi01] Stanislav Smirnov, Critical percolation in the plane: Conformal invariance, Cardy's formula, scaling limits, C. R. Acad. Sci. Paris Sér. I Math. 333 (2001), no. 3, 239-244. MR.1851632 (2002f:60193)

[Zif95] Robert M. Ziff, Proof of crossing formula for $2 D$ percolation, J. Phys. A 28 (1995), no. 22, 6479-6480. MR1379947 (96m:82030)

Department of Mathematics, University of Washington, Box 354350, Seattle, WashINGTON 98195

Microsoft Research, One Microsoft Way, Redmond, Washington 98052 